\title{
Hadron beam evolution in microbunched electron cooling
}

\author{
P. Baxevanis and G. Stupakov (1) \\ SLAC National Accelerator Laboratory Menlo Park, California 94025, USA
}

(Received 23 June 2020; accepted 19 October 2020; published 6 November 2020)

\begin{abstract}
The technique of microbunched electron cooling (MBEC) is a coherent cooling scheme with possible applications in high-energy hadron and electron-ion machines. In our previous work we analyzed the cooling of the hadron energy spread and transverse emittance using a one-dimensional (1D) technique that tracked the microscopic fluctuations in the hadron and electron beams. However, in order to obtain analytical expressions for our key quantities, we limited ourselves to calculating and optimizing only the initial values of the cooling rates. In this paper, we extend our approach so that it properly addresses the issue of the long-term, dynamic evolution of the hadron beam. In order to do so, it becomes necessary to consider the synchrotron motion of the hadron beam, in conjunction with the effects of diffusion and intrabeam scattering (IBS). With these modifications, our formalism allows us to develop a simple numerical tool that can effectively model the final state of hadron beam after many passages through the MBEC cooler.
\end{abstract}

DOI: 10.1103/PhysRevAccelBeams.23.111001

\section{INTRODUCTION}

In order to achieve the challenging brightness requirements for the hadron beam in prospective hadron-hadron or electron-ion circular colliders, various coherent cooling schemes have been proposed as a possible solution [1,2]. In all these techniques, random fluctuations in the hadron beam lead to an energy modulation being imprinted onto a co-propagating electron beam through the space charge interaction. This energy modulation is then converted into density modulation (bunching) by passage through a dispersive chicane and this bunching is subsequently amplified by means of a suitable mechanism. Finally, the cooler electron beam is once more copropagated with the hadrons and interacts with them via the boosted space charge field. If this process is repeated over many times, the brightness of the hadron beam can be enhanced significantly (cooling), for both the longitudinal and the transverse degrees of freedom. While other coherent cooling schemes make use of the free-electron-laser (FEL) effect [2], in microbunched electron cooling (MBEC, proposed in [3]) one relies on the principle of the space charge amplifier in order to boost the bunching of the cooler electron beam: the plasma oscillations induced in the electron beam during its passage through a drift stage result in energy modulation, which is then translated into enhanced density

Published by the American Physical Society under the terms of the Creative Commons Attribution 4.0 International license. Further distribution of this work must maintain attribution to the author(s) and the published article's title, journal citation, and DOI. modulation in a subsequent chicane. Originally conceived for purposes of coherent radiation generation [4], the space charge amplifier method provides a broadband alternative to narrow-band amplification techniques such as the FEL.

In our earlier work [5-7], we utilized a one-dimensional (1D), Vlasov equation-based technique in order to obtain useful, analytical expressions for the MBEC cooling rates (both transverse and longitudinal). However, in the process of doing so, we limited ourselves to calculating and optimizing only the initial values of the above-mentioned cooling rates (i.e., those achieved at the beginning of the cooling). In this paper, we build on our previous results and extend our approach so that it accurately covers the longterm, self-consistent evolution of the hadron beam. In order to do so, it becomes essential to consider the synchrotron motion induced by the rf system of the ring, which causes the hadrons to move relative to each other in the longitudinal direction. Along with synchrotron oscillations, we also need to take into account other important effects such as diffusion and intrabeam scattering (IBS). With these modifications, our formalism enables us to develop a kinetic equation for the cooling process, which leads to an efficient numerical tool that can model the evolution of the hadron beam over an arbitrary number of passages through the cooler. A similar study for the Coherent Electron Cooling has been recently published by G. Wang in Ref. [8].

This paper is organized as follows: in Sec. II, we introduce the basic concepts regarding the synchrotron motion of the hadrons (including the action-angle variables) and review the averaging process that leads to the kinetic equation for the hadron beam. In Sec. III, we present the details of this kinetic equation for a typical MBEC 


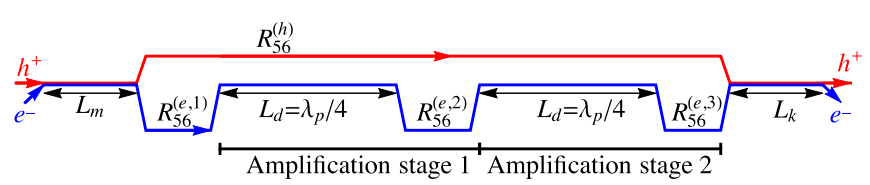

FIG. 1. MBEC configuration with two amplification stages (the length $L_{d}$ is a free parameter but, in practice, its value is $\sim \lambda_{p}$, where $\lambda_{p}$ is the wavelength of plasma oscillations in the electron beam).

configuration and also show some preliminary results from its numerical solution. In Sec. IV we show how to incorporate IBS into our analysis and demonstrate how the deleterious effects of the latter can be effectively counterbalanced by an MBEC cooler for a machine like eRHIC. Finally, Sec. V summarizes the main results of this study.

\section{PRELIMINARIES}

In MBEC, the hadron beam first imprints an energy modulation on the copropagating (cooler) electron beam in a segment of the machine known as the modulator. This energy modulation is then converted into a density modulation (bunching) after the e-beam passes through a dispersive chicane section with strength $R_{56}^{(e, 1)}$ (Fig. 1). In the meantime, the hadrons are transported through their ownseparate-section of the lattice, which also includes a chicane with strength $R_{56}^{(h)}$. The bunched electron beam then once again interacts with the hadrons in a subsequent section of the machine (the kicker), in a way that can ultimately lead to a significant reduction in the hadron energy spread and transverse emittance, after many passages through the cooling section. In order to accelerate this process and ensure that the cooling timescale is small enough for practical purposes, additional amplification stages are typically required, in which the bunching of the electron beam is boosted through the space charge effect. Each such amplification stage consists of a drift space followed by a chicane of strength $R_{56}^{(e, j)}$ ( $j=2, \ldots, M+1$, where $M$ is the total number of stages). For simplicity, in this paper we assume that all stages have the same length $L_{d}$. To simplify our analysis, we will neglect transverse cooling effects throughout this paper. Hence, the appropriate starting point for our treatment is the kinetic equation for the $1 \mathrm{D}$ hadron distribution function $F_{h}(\eta, t)$ in the presence of longitudinal cooling and diffusion, where $\eta=E / E_{0}-1$ is the relative energy deviation variable for the hadrons. We can readily obtain this particular form of the kinetic equation from the results of our previous analysis, namely Eq. (41) of Ref. [7]. To ignore the transverse cooling effects we set $R, S \rightarrow 0$ in that equation to obtain

$\frac{\partial F_{h}}{\partial t}=D_{\eta} \frac{\partial^{2} F_{h}}{\partial \eta^{2}}+\frac{r_{h} c}{2 \pi \gamma T} \frac{\partial}{\partial \eta}\left(F_{h} \int_{-\infty}^{\infty} d k \mathcal{Z}(k)\left\{e^{i k R_{56}^{(h)} \eta}-1\right\}\right)$, where $\gamma=E_{0} / m_{h} c^{2}$ is the relativistic factor (common for electrons and hadrons), $T$ is the revolution period for the ring, $r_{h}=(Z e)^{2} / m_{h} c^{2}$ is the classical radius of the hadrons and the diffusion coefficient $D_{\eta}$ can be expressed in terms of the impedance $\mathcal{Z}(k)$ by Eq. (35) of [7]. The MBEC impedance is, in turn, expressed analytically by Eq. (48) of [7], appropriately modified by the gain factors for a cascade of amplification stages. For a detailed derivation of the latter, we refer the interested reader to the analysis of Ref. [6]. We should also note that the diffusion coefficient $D_{\eta}$ is outside the partial derivative as it does not depend on the energy variable. Moreover, we clarify that Eq. (1) has been specifically derived for the case of MBEC and cannot be directly applied to other forms of cooling, such as stochastic cooling. That being said, the fluctuations-based approach on which our derivation is based has been used in the past by one of the authors (GS) for treating stochastic cooling in terms of a simple model that is typically found in textbooks [9].

However, this form of the kinetic equation only applies to the distribution function in the so-called local approximation, that is when the dependence of $F_{h}$ with respect to the intra-beam position $z_{b}$ is not taken into consideration. Moreover, it does not quantify the impact of the synchrotron oscillations imposed on the hadrons by the RF system of the ring, which change their relative longitudinal positions within the beam. If one wishes to incorporate these two interconnected effects, the kinetic equation becomes

$$
\begin{aligned}
& \frac{\partial F_{h}}{\partial t}-c \alpha \eta \frac{\partial F_{h}}{\partial z_{b}}+\frac{\omega_{s}^{2}}{\alpha c} z_{b} \frac{\partial F_{h}}{\partial \eta} \\
& =D_{\eta}\left(z_{b}\right) \frac{\partial^{2} F_{h}}{\partial \eta^{2}}+\frac{r_{h} c}{2 \pi \gamma T} \frac{\partial}{\partial \eta}\left(F_{h} \int_{-\infty}^{\infty} d k \mathcal{Z}\left(k, z_{b}\right)\left\{e^{i k R_{56}^{(h)} \eta}-1\right\}\right),
\end{aligned}
$$

where $F_{h}=F_{h}\left(z_{b}, \eta, t\right)$. Here, $\omega_{s}$ is the synchrotron oscillation frequency, while $\alpha$ is the (dimensionless) momentum compaction factor. This modified form of the kinetic equation is justified by the fact that-in the absence of collective effects such as cooling, wakefields etc-the linearized, longitudinal equations of motion for the hadrons are $d z_{b} / d t=-c \alpha \eta$ and $d \eta / d t=\omega_{s}^{2} z_{b} / \alpha c$. More details regarding the theory and nomenclature of synchrotron oscillations can be found in [10]. We emphasize that the $z_{b}$-dependence in the impedance and the diffusion coefficient is a consequence of the finite longitudinal size of the cooler and hadron beams, which results in a $z_{b}$-dependence for their two respective currents. Next, if we introduce the scaled quantities

$$
\tilde{t}=t / \tau_{s} \equiv \omega_{s} t, \quad z=-\omega_{s} z_{b} / c \alpha,
$$

the kinetic equation becomes 
$\frac{\partial F_{h}}{\partial \tilde{t}}+\eta \frac{\partial F_{h}}{\partial z}-z \frac{\partial F_{h}}{\partial \eta}=D(z) \frac{\partial^{2} F_{h}}{\partial \eta^{2}}+\frac{1}{2} \frac{\partial}{\partial \eta}\left(\nu(z, \eta) F_{h}\right)$,

where $D=\tau_{s} D_{\eta}$ and

$$
\frac{\nu}{2}=\tau_{s} \frac{r_{h} c}{2 \pi \gamma T} \int_{-\infty}^{\infty} d k \mathcal{Z}(k, z)\left\{e^{i k k_{56}^{(h)} \eta}-1\right\} .
$$

The cooling timescale is typically much longer than the synchrotron oscillation period. As a result, the kinetic equation should be properly averaged over the synchrotron oscillations. In order to accomplish this task, we first introduce the action-angle variables $(J, \phi)$ via the transformation

$$
z=\sqrt{2 J} \cos \phi, \quad \eta=\sqrt{2 J} \sin \phi,
$$

the inverse of which is

$$
J=\frac{1}{2}\left(z^{2}+\eta^{2}\right), \quad \tan \phi=\frac{\eta}{z} .
$$

Next, we need to express the distribution function $F_{h}$ in terms of $J, \phi$ and $\tilde{t}$, transform the Vlasov equation to these new variables and perform an averaging over $\phi$. This manipulation is carried out in Appendices A and B. The final result is an evolution equation for the averaged distribution function $\bar{F}_{h}(J, \tilde{t})$, namely

$$
\frac{\partial \bar{F}_{h}}{\partial \tilde{t}}=\frac{1}{\sqrt{2}} \frac{\partial}{\partial J}\left(\sqrt{J} \bar{\nu}(J) \bar{F}_{h}\right)+\frac{\partial}{\partial J}\left(\bar{D}(J) J \frac{\partial \bar{F}_{h}}{\partial J}\right)
$$

where the average cooling/diffusion functions $\bar{\nu}(J)$ and $\bar{D}(J)$ are given by Eqs. (A9) and (B11), respectively.

\section{KINETIC EQUATION}

In the absence of cooling and diffusion, the (equilibrium) distribution function of the hadrons is a function of $J$ alone. From the definition of the longitudinal action-angle variables, we recall that

$$
J=\frac{1}{2}\left(\eta^{2}+z^{2}\right)=\frac{1}{2}\left(\eta^{2}+\frac{\omega_{s}^{2}}{\alpha^{2} c^{2}} z_{b}^{2}\right) .
$$

With this relation in mind, we consider an initial distribution of the form

$$
\bar{F}_{h}(J, \tilde{t}=0) \propto \exp \left(-\frac{J}{\sigma_{h, 0}^{2}}\right)=\exp \left(-\frac{z_{b}^{2}}{2\left(\sigma_{z, 0}^{(h)}\right)^{2}}-\frac{\eta^{2}}{2 \sigma_{h, 0}^{2}}\right) .
$$

This particular choice corresponds to a hadron beam with a Gaussian current/energy profile, its parameters satisfying the matching condition

$$
\frac{\omega_{s}^{2}}{\alpha^{2} c^{2}}\left(\sigma_{z, 0}^{(h)}\right)^{2}=\sigma_{h, 0}^{2}
$$

If the initial hadron bunch length $\sigma_{z, 0}^{(h)}$ and rms energy spread $\sigma_{h, 0}$ satisfy Eq. (11), the hadron distribution does not evolve in time when cooling and diffusion are absent.

To facilitate the eventual numerical solution of the kinetic equation we further define the scaling $\tilde{J}=J / \sigma_{h, 0}^{2}$ and $\tilde{\eta}=\eta / \sigma_{h, 0}$, so that $\tilde{\eta}=\sqrt{2 \tilde{J}} \sin \phi$. In terms of these new energy and action variables, we have $\bar{F}_{h}(\tilde{t}=0) \propto$ $\exp (-\tilde{J})$ and the scaled kinetic equation becomes

$$
\frac{\partial \bar{F}_{h}}{\partial \tilde{t}}=\frac{\partial}{\partial \tilde{J}}\left(\tilde{\nu}(\tilde{J}) \bar{F}_{h}\right)+\frac{\partial}{\partial \tilde{J}}\left(\tilde{J} \tilde{D}(\tilde{J}) \frac{\partial \bar{F}_{h}}{\partial \tilde{J}}\right) .
$$

Here, the cooling function $\tilde{\nu}(\tilde{J})$ is given by [6]

$$
\begin{aligned}
\tilde{\nu}(\tilde{J}) \equiv & \frac{\sqrt{2}}{\sigma_{h, 0}} \frac{\bar{\nu}}{2} \sqrt{\tilde{J}}=\frac{\tau_{s}}{2 \pi T} \int_{-\pi}^{\pi} d \phi \sin \phi \frac{4 \sqrt{2} I_{e}(\tilde{J}, \phi) L_{m} L_{k} r_{h}}{\Sigma^{3} \pi \gamma^{3} I_{A} \sigma_{e} \sigma_{h, 0}} \times(-1)^{M} q_{e, 1} q_{e, 2} \ldots q_{e, M+1} \\
& \times\left(\frac{1}{\sigma_{e}} \sqrt{\frac{2 I_{e}(\tilde{J}, \phi)}{\gamma I_{A}}}\right)^{M} \int_{0}^{\infty} d \varkappa \varkappa\left(\frac{\varkappa H\left(r_{p} \varkappa, 1\right)}{r_{p}}\right)^{M / 2} \exp \left(-\varkappa^{2}\left(q_{e, 1}^{2}+q_{e, 2}^{2}+\cdots+q_{e, M+1}^{2}\right) / 2\right) \\
& \times \sqrt{\tilde{J}} \sin \left(\varkappa q_{h} \sqrt{2 \tilde{J}} \sin \phi\right) H^{2}(\varkappa, r) \sin ^{M}\left(r_{p} \frac{\Omega_{p}(\tilde{J}, \phi) L_{d}}{c} \sqrt{\frac{2 \varkappa H\left(r_{p} \varkappa, 1\right)}{r_{p}}}\right),
\end{aligned}
$$

where we have used the analytical expression for the MBEC impedance, assuming a cascade of $M$ amplification stages with equal length $L_{d}$. Specifically, $L_{m}$ and $L_{k}$ are the lengths of the modulator and kicker sections, $\sigma_{e}$ is the rms energy spread of the electrons (assuming a fixed, Gaussian energy profile for the cooler) and $I_{A}=m_{e} c^{3} / e \approx 17 \mathrm{kA}$ is the Alfven current. We assume that the electron beam current has a longitudinal profile $I_{e}\left(z_{e}\right)$; when this current overlaps with the hadron beam in the modulator and the kicker the coordinate $z_{e}$ can be identified with $z_{b}$ and then expressed through $\tilde{J}, \phi$, as indicated in the argument of $I_{e}$ in Eq. (13). As far as the transverse properties of the two 
beams are concerned, we adopt Gaussian profiles for both and assume that (a) at the modulator and kicker, the interacting beams have an identical, elliptical cross section characterized by a horizontal rms size $\Sigma$ and a size aspect ratio $r$ (b) at the amplification stages, the e-beam is round with a common rms size $r_{p} \Sigma$. The squeeze factor $r_{p}$ is also involved in the definition of the plasma frequency $\Omega_{p}$, which is given by $\Omega_{p}=\left(c / r_{p} \Sigma\right)\left(I_{e} / \gamma^{3} I_{A}\right)^{1 / 2}$. Moreover, $q_{h}=R_{56}^{(h)} \sigma_{h, 0} \gamma / \Sigma$ is the scaled hadron chicane strength and $q_{e, j}=R_{56}^{(e, j)} \sigma_{e} \gamma / \Sigma$ are the normalized strengths of the various electron chicanes. Lastly, the important function $H(\hat{k}, r)$, which is directly related to the Fourier transform of the space charge interaction function, is defined by $H(\hat{k}, r)=\hat{k} \int_{0}^{\infty} d \tau \tau \exp \left(-\hat{k}^{2} \tau^{2} / 4\right) / \sqrt{\left(\tau^{2}+4\right)\left(\tau^{2}+4 r^{2}\right)}$. The only additional clarification necessary is the following: for a cooler beam current of the form $I_{e}=I_{e, 0} \exp \left(-z_{b}^{2} / 2\left(\sigma_{z, 0}^{(e)}\right)^{2}\right)$-fixed and centered around $z_{b}=0$, with $\sigma_{z, 0}^{(e)}$ and $I_{e, 0}$ being the electron $\mathrm{rms}$ bunch length and peak current, respectively-transforming into the scaled action-angle variables yields

$$
I_{e}(\tilde{J}, \phi)=I_{e, 0} \exp \left(-\left(\sigma_{z, 0}^{(h)} / \sigma_{z, 0}^{(e)}\right)^{2} \tilde{J} \cos ^{2} \phi\right) .
$$

This function is to be substituted into the formula for the plasma frequency, yielding an analytical expression for $\Omega_{p}(\tilde{J}, \phi)$.

As far as the diffusion function $\tilde{D}(\tilde{J})$ is concerned, its definition is

$$
\tilde{D}(\tilde{J}) \equiv \frac{\bar{D}}{\sigma_{h, 0}^{2}}=\frac{1}{\pi} \int_{-\pi}^{\pi} d \phi \frac{\tau_{s} D_{\eta}(\tilde{J}, \phi)}{\sigma_{h, 0}^{2}} \sin ^{2} \phi .
$$

Assuming (for simplicity's sake) that the diffusion coefficient $D_{\eta}$ is dominated by the hadron noise, we can calculate it using Eq. (3) from [11], namely

$$
\begin{aligned}
D_{\eta}^{(h)}(\tilde{J}, \phi)= & \frac{4 \sigma_{h, 0}^{2}}{\pi T} \frac{I_{h} I_{e}^{2}(\tilde{J}, \phi) r_{h}^{2} L_{m}^{2} L_{k}^{2}}{I_{A}^{3} \gamma^{7} r_{e} \Sigma^{5} \sigma_{e}^{2} \sigma_{h, 0}^{2}} \times \frac{1}{\sigma_{e}^{2 M}}\left(\frac{2 I_{e}(\tilde{J}, \phi)}{\gamma I_{A}}\right)^{M} \\
& \times q_{e, 1}^{2} q_{e, 2}^{2} \ldots q_{e, M+1}^{2} \int_{-\infty}^{\infty} d \varkappa \varkappa^{2} H^{4}(\varkappa, r) \exp \left(-\left(q_{e, 1}^{2}+q_{e, 2}^{2}+\cdots+q_{e, M+1}^{2}\right) \varkappa^{2}\right) \\
& \times\left(\frac{\varkappa H\left(r_{p} \varkappa, 1\right)}{r_{p}}\right)^{M} \sin ^{2 M}\left(r_{p} \frac{\Omega_{p}(\tilde{J}, \phi) L_{d}}{c} \sqrt{\frac{2 \varkappa H\left(r_{p} \varkappa, 1\right)}{r_{p}}}\right) .
\end{aligned}
$$

Of course, this approximation neglects effects due to the shot noise in the electron beam itself, which could-in fact-be significant when hadron and electron currents are similar. Including electron noise effects in our analysis would be straightforward, the main difference being a modification of the diffusion profile $\tilde{D}(\tilde{J})$.

One final complication remains to be addressed: the hadron current $I_{h}=I_{h}(z, \tilde{t})$ depends on the evolving distribution function $\bar{F}_{h}(J, \tilde{t})$ and therefore is an unknown quantity. In general this also makes the diffusion coefficient $D_{\eta}^{(h)}$ a function of $\tilde{J}, \phi$ and $\tilde{t}$. However, we can simplify the problem by assuming that, throughout the evolution process (cooling or heating), the hadron current profile stays centered around $z=0$ and remains much broader than the fixed electron current profile $I_{e}(z), \sigma_{z, 0}^{(h)} \gg \sigma_{z, 0}^{(e)}$. This allows us to replace $I_{h}(z, \tilde{t})$ by its value at the location of the electron beam, $I_{h}(0, \tilde{t})$, in the equation given above, which takes this quantity outside the $\phi$-integral. Still, we need to figure out what this peak hadron current is in terms of $\tilde{t}$. Given that the hadron current profile satisfies $I_{h}(z, \tilde{t}) \propto \int_{-\infty}^{\infty} d \eta F_{h}(z, \eta, \tilde{t}) \propto \int_{-\infty}^{\infty} d \eta \bar{F}_{h}\left[\left(\eta^{2}+z^{2}\right) / 2, \tilde{t}\right]$, we can show that
$I_{h}(z, \tilde{t})=I_{h, 0} \frac{\int_{0}^{\infty} d \eta \bar{F}_{h}\left[\left(\eta^{2}+z^{2}\right) / 2, \tilde{t}\right]}{\int_{0}^{\infty} d \eta \bar{F}_{h}\left(\eta^{2} / 2,0\right)}=I_{h, 0} \xi(\tilde{t}) \chi(z, \tilde{t})$,

where

$$
\xi(\tilde{t})=\frac{\int_{0}^{\infty} d \eta \bar{F}_{h}\left(\eta^{2} / 2, \tilde{t}\right)}{\int_{0}^{\infty} d \eta \bar{F}_{h}\left(\eta^{2} / 2,0\right)}
$$

is the compression factor for the hadron peak current,

$$
\chi(z, \tilde{t})=\frac{\int_{0}^{\infty} d \eta \bar{F}_{h}\left[\left(\eta^{2}+z^{2}\right) / 2, \tilde{t}\right]}{\int_{0}^{\infty} d \eta \bar{F}_{h}\left(\eta^{2} / 2, \tilde{t}\right)},
$$

is the scaled current profile and $I_{h, 0}$ is the initial value of the peak current. Using the latter, we find that the time dependence in the diffusion coefficient factors out, and we can calculate and tabulate a diffusion function $\tilde{D}_{0}(\tilde{J})$, which along with the cooling function $\tilde{\nu}(\tilde{J})$, only depends on the fixed parameters of the system. Since $I_{h}(0, \tilde{t})=I_{h, 0} \xi(\tilde{t})$, the kinetic equation for $\bar{F}_{h}(\tilde{J}, \tilde{t})$ can be rewritten as 

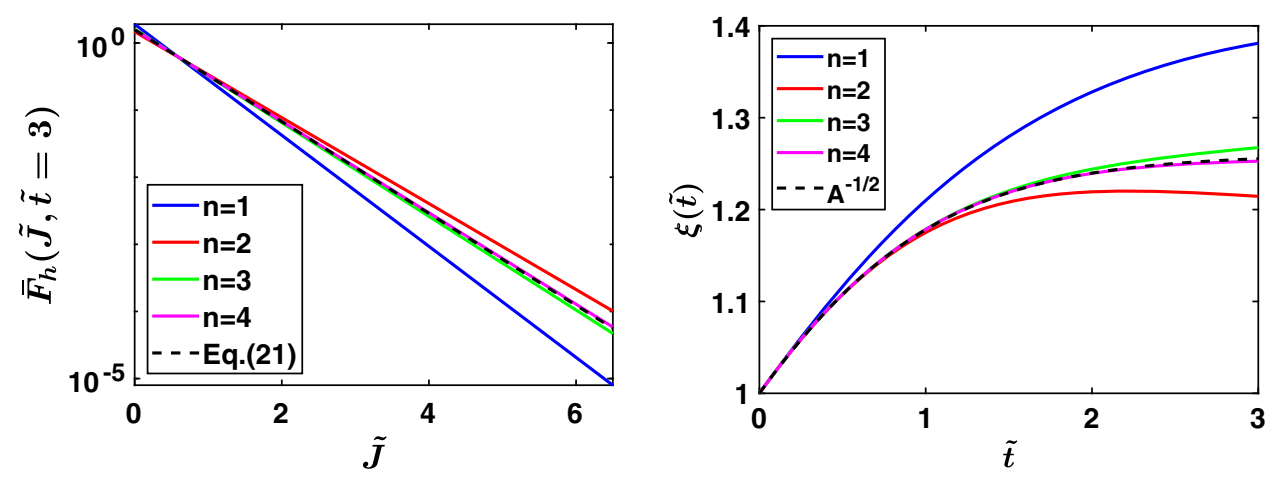

FIG. 2. Iterative numerical solution (colored plots) versus the analytical solution (dashed lines), for the distribution function (left) and the $\xi$-factor (right), assuming a test case with $\nu_{0}=1.0$ and $D_{0}=0.5$ (the y-axis of the left plot is logarithmic). Here, $n$ is the integer iteration index, and adequate convergence is achieved after four iterations.

$$
\frac{\partial \bar{F}_{h}}{\partial \tilde{t}}=\frac{\partial}{\partial \tilde{J}}\left(\tilde{\mathcal{L}}(\tilde{J}) \bar{F}_{h}\right)+\xi(\tilde{t}) \frac{\partial}{\partial \tilde{J}}\left(\tilde{J} \tilde{D}_{0}(\tilde{J}) \frac{\partial \bar{F}_{h}}{\partial \tilde{J}}\right)
$$

which, for a given function $\xi(\tilde{t})$, is a form that can be processed by the built-in PDE solver of Matlab. Of course, since $\xi$ itself depends on the unknown distribution $\bar{F}_{h}$, the numerical solution needs to be implemented in an iterative fashion: first, the time dependence of the hadron current profile is neglected, which means that $\xi(\tilde{t})=1$ and Eq. (20) is solved with this assumption in mind. Using the obtained solution for $\bar{F}_{h}$ one then calculates $\xi$ as a function of $\tilde{t}$ via Eq. (18) and accordingly updates the solution of Eq. (20), repeating this loop until the required degree of convergence is achieved.

To benchmark this iterative process, it is instructive to point out that, in the special case with $\tilde{\nu}(\tilde{J})=\nu_{0} \tilde{J}$ and $\tilde{D}_{0}(\tilde{J})=D_{0}$ (where $\nu_{0}$ and $D_{0}$ are constants), Eq. (20) has an analytical solution expressed by

$$
\bar{F}_{h}(\tilde{J}, \tilde{t})=\frac{1}{A(\tilde{t})} \exp \left(-\frac{\tilde{J}}{A(\tilde{t})}\right),
$$

where

$$
A(\tilde{t})=\left\{\frac{D_{0}}{\nu_{0}}\left(1-e^{-3 \nu_{0} \tilde{t} / 2}\right)+e^{-3 \nu_{0} \tilde{t} / 2}\right\}^{2 / 3} .
$$

Figure 2 shows an illustration of the gradual convergence of the numerical solution toward its analytical counterpart. Apart from the distribution function itself, the other metric used in this example is the compression factor $\xi$, which can be shown to be equal to $A^{-1 / 2}$ for the analytically tractable test case under consideration.

Having verified the soundness of the iterative technique, we consider an MBEC configuration for eRHIC using the parameter set of Table I. Two amplification sections with a total length of about $30 \mathrm{~m}$ were assumed in this simulation, along with a $1 \mathrm{nC}$ cooler electron bunch. In Fig. 3 we plot the actual MBEC cooling/diffusion functions for these parameters, demonstrating that they are markedly different than the simple demonstration case discussed earlier. Indeed the diffusion function is characterized by a sharp peak around the origin, followed by a much more gradual decrease with $\tilde{J}$. Moreover, we observe that the cooling function actually becomes negative for $\tilde{J}>4$. As we shall see later on, this leads to heating of hadrons with relatively large values of action. The main results of the numerical solution of the kinetic equation are summarized in Fig. 4. The upper right sub-plot of this figure shows the temporal evolution of the hadron peak current $I_{p} \equiv I_{h}(0, t)$ (blue curve) and the longitudinal emittance

$$
\epsilon \equiv\langle J\rangle=\int_{0}^{\infty} d J J \bar{F}_{h}(J, t)
$$

TABLE I. Parameters of the eRHIC collider with a hypothetical MBEC cooler.

\begin{tabular}{lc}
\hline \hline Common relativistic factor, $\gamma$ & 293 \\
Proton/electron energy $\gamma m_{h} c^{2} / \gamma m_{e} c^{2}[\mathrm{GeV}]$ & $275 / 0.15$ \\
Proton/electron rms energy spread, & $4.6 \times 10^{-4} / 1 \times 10^{-4}$ \\
$\quad \sigma_{h, 0} / \sigma_{e}$ & \\
Proton/electron rms bunch length, & $5 / 0.4$ \\
$\quad \sigma_{z, 0}^{(h)} / \sigma_{z, 0}^{(e)}$ [cm] & $23 / 30$ \\
Proton/electron peak current, $I_{h, 0} / I_{e, 0}[\mathrm{~A}]$ & $1.3 \times 10^{-5}$ \\
Ring revolution period $T$ [s] & 50 \\
Modulator and kicker lengths $L_{m}, L_{k}[\mathrm{~m}]$ & 15 \\
Amplification stage drift length $L_{d}[\mathrm{~m}]$ & 2 \\
Number of amplification stages & 700 \\
Proton beam horizontal size $\Sigma[\mu \mathrm{m}]$ & 0.60 \\
x-y proton beam aspect ratio & 0.20 \\
e-beam squeeze factor & $1.7 / 5.1$ \\
Proton/electron chicane strength & \\
$\quad R_{56}^{(h)} / R_{56}^{(e)}$ [mm] & \\
\hline \hline
\end{tabular}



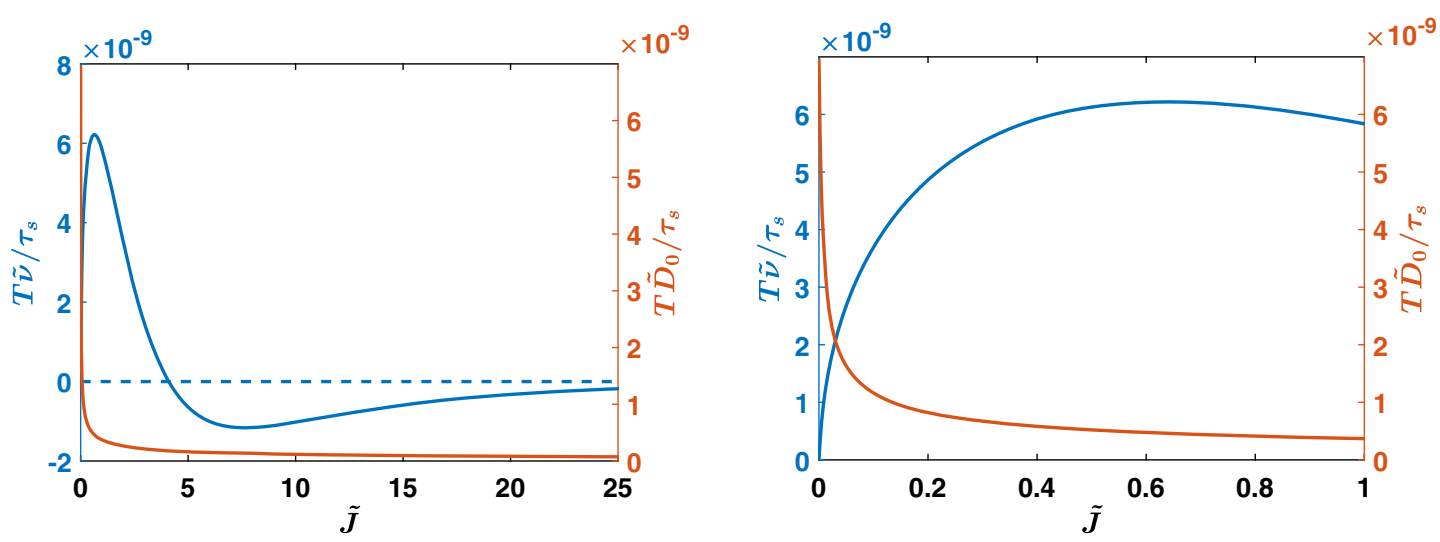

FIG. 3. Plots of the cooling and diffusion functions versus the scaled synchrotron action $\tilde{J}$ (data for the eRHIC parameter set). The right subplot focuses on cooling and diffusion for small action values.
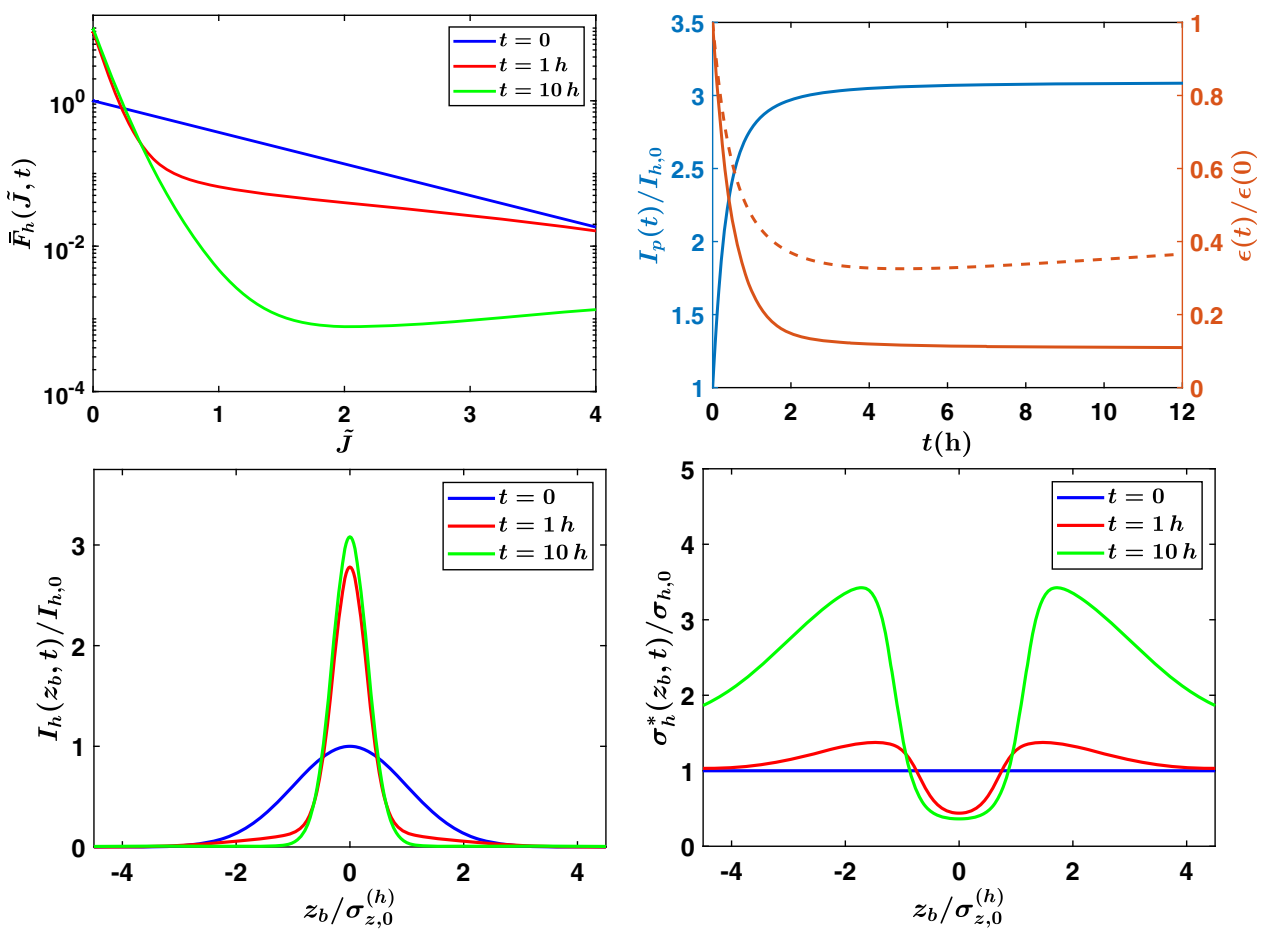

FIG. 4. Upper right: proton peak current (blue), total longitudinal emittance (brown-dashed) and 95\% emittance (brown) as functions of time. For the remainder, moving counterclockwise, we plot the proton distribution function, current and local energy spread profiles at different times (data for the eRHIC parameter set).

the latter shown in the brown dashed curve. In fact, the ratio of $\epsilon$ to its initial value $\epsilon_{0} \equiv \epsilon(0)$ can also be used to extract the rms energy spread $\sigma_{h}$ and bunch length $\sigma_{z}^{(h)}$ of the evolving hadron beam via the relation $\sigma_{h} / \sigma_{h, 0}=\sigma_{z}^{(h)} / \sigma_{z, 0}^{(h)}=\sqrt{\epsilon / \epsilon_{0}}$. Thus, a reduction in the value of the longitudinal emittance is accompanied not only by cooling (i.e., a decrease in $\sigma_{h}$ ) but also by a corresponding shortening of the hadron bunch, at least in an rms sense. The latter feature also results in an increase of the bunch peak current, as is evident from the blue curve. Of course, this increase in the hadron current leads to intensified diffusion effects that oppose further cooling, eventually resulting in a rough stabilization (though not a steady-state in the exact, mathematical sense of the term). As defined above, the longitudinal emittance $\epsilon$ encompasses the entire hadron beam. In practice one may also wish to redefine the emittance using only that part of the beam that contains a specified fraction of the total charge. In our case we have also calculated the emittance for that portion of the bunch that contains $95 \%$ of the proton charge (solid brown curve). As this modified definition excludes the tail ends of the beam, the fractional emittance is smaller 


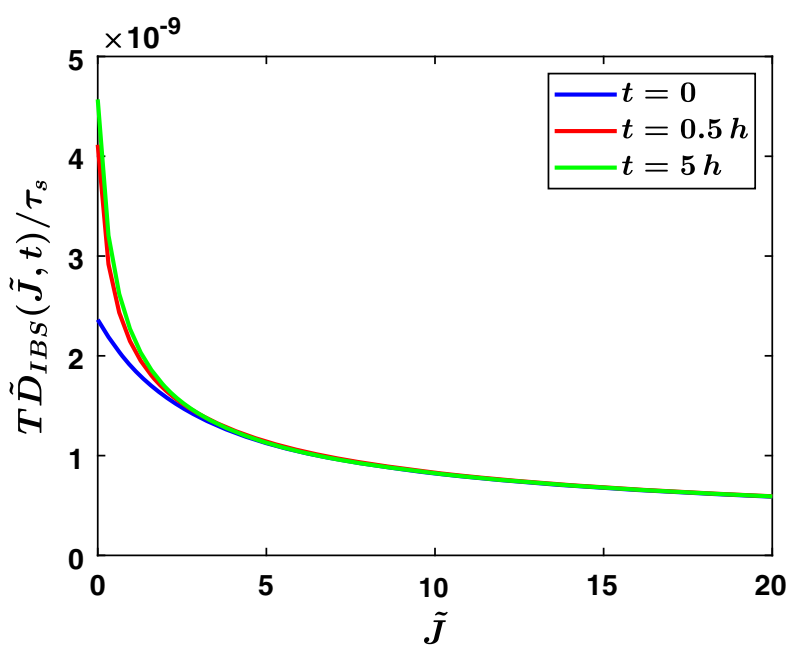

FIG. 5. IBS diffusion term versus the scaled synchrotron action $\tilde{J}$ at different times (data for the eRHIC parameter set, assuming both cooling and IBS).

than the one for the entire bunch. Moreover, being a quantity that largely excludes the tail regions of the distribution, the $95 \%$ emittance is less sensitive than the total rms emittance with respect to the tail heating effect discussed below.

Moving counterclockwise, the remaining subfigures of Fig. 4 show snapshots of the proton distribution function, as well as the current and local energy spread profiles at different times. The last quantity is defined by $\sigma_{h}^{*}(z, t)^{2} \propto$ $\int_{0}^{\infty} d \eta \eta^{2} \bar{F}_{h}\left(\left(\eta^{2}+z^{2}\right) / 2, t\right)$ and is used as a measure of the local value of the energy spread along the bunch. It is evident that both the proton distribution function and the current profile exhibit a gradual sharpening trend as a result of the cooling effect. At the same time, the right lower subfigure shows that, while the core part of the beam (i.e., the one containing most of the current) undergoes cooling, there is considerable heating at the tail ends of the bunch, a tendency that becomes more and more pronounced with time. This tail heating effect explains why, in contrast to the fractional emittance and peak current (both of which are roughly stabilized after the first four hours), the total emittance starts to increase at about that time. In experimental practice this secondary heating effect should be kept under control since, when coupled with dispersion, it can generate deleterious beam halos.
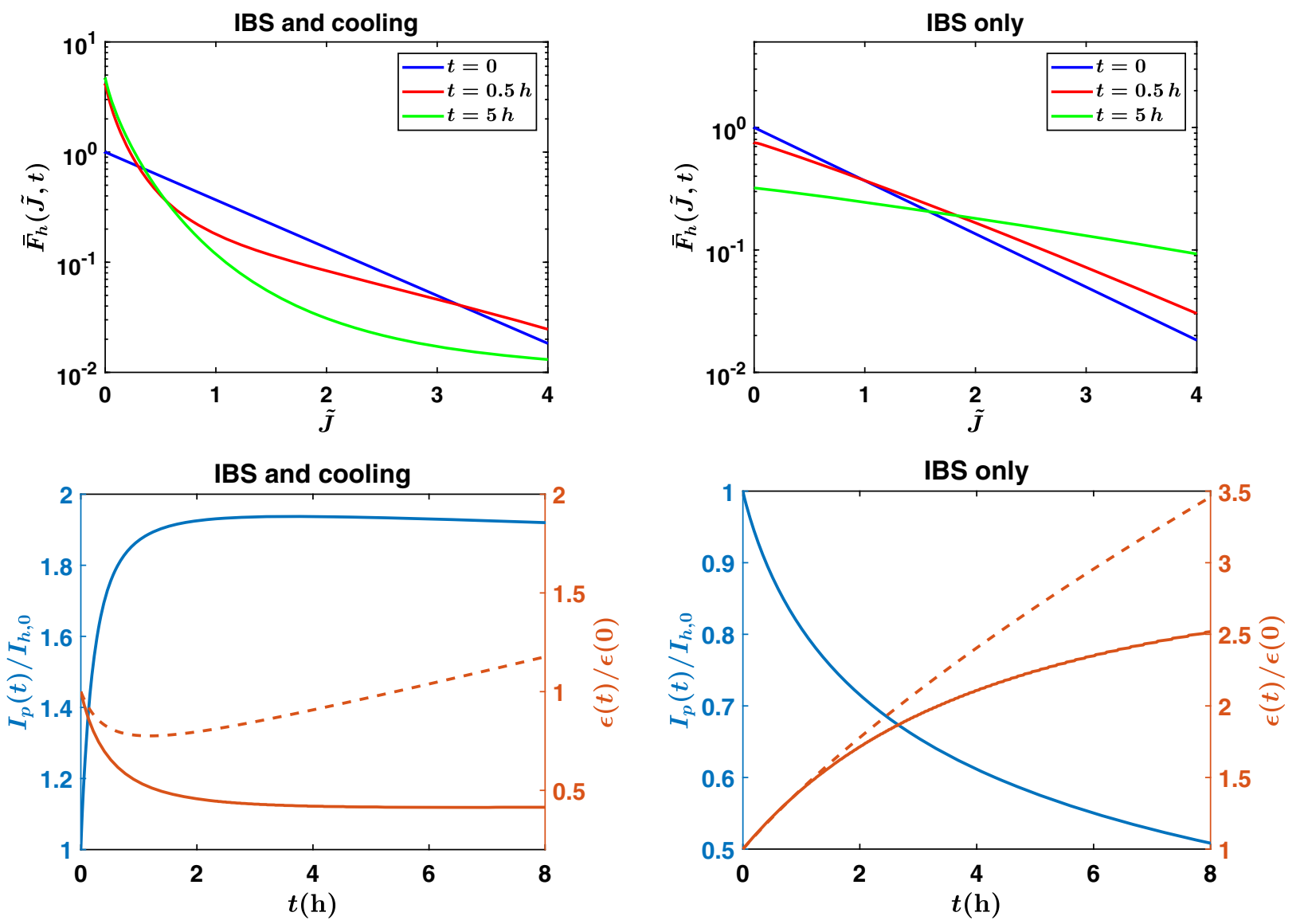

FIG. 6. Upper panel: hadron distribution function at selected times during the cooling process. Lower panel: hadron peak current (blue curve), total longitudinal emittance (dashed brown curve) and core part (95\%) emittance (solid brown curve) versus time. The data shown compare and contrast the cases with and without cooling. 

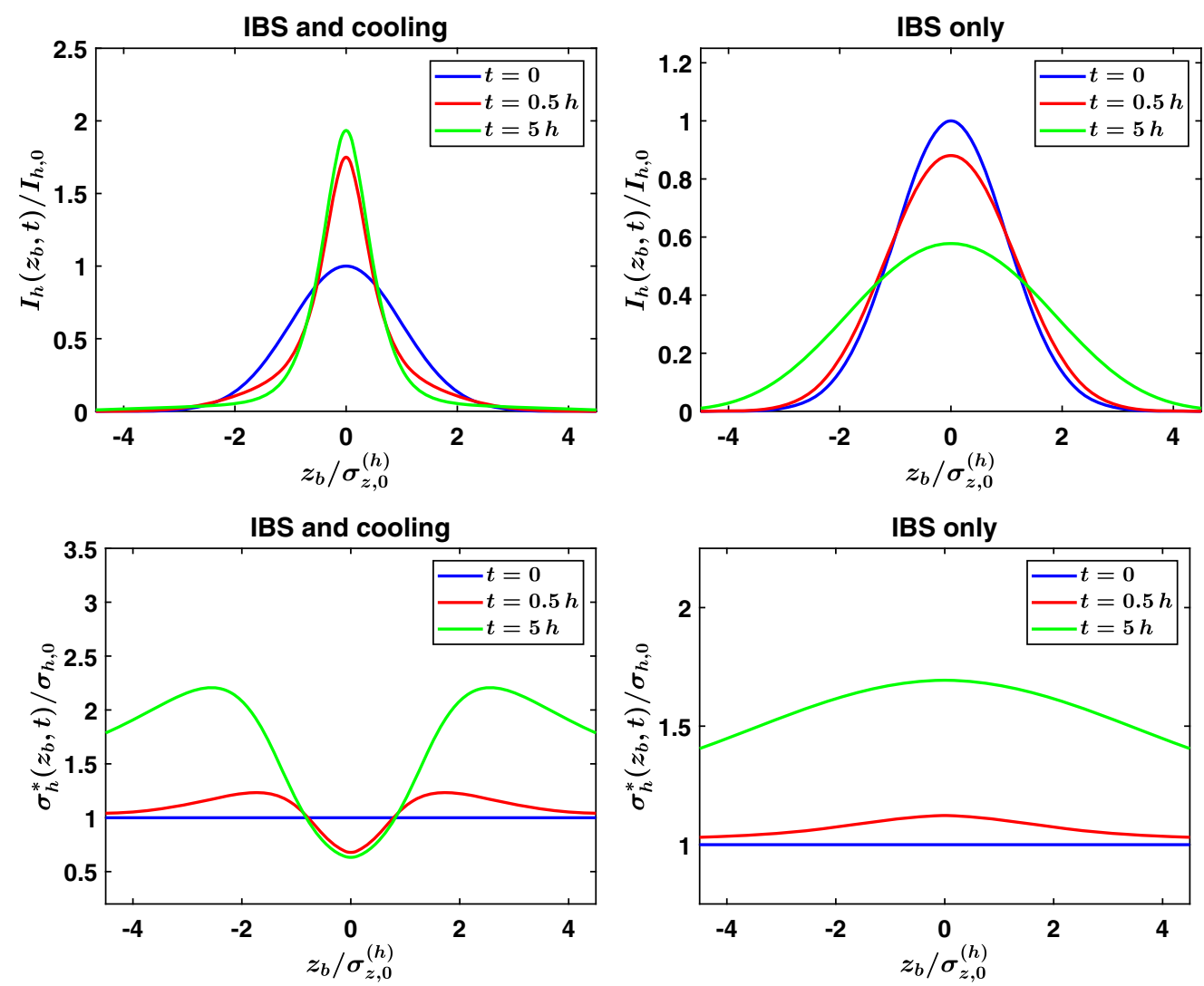

FIG. 7. Upper panel: hadron current profiles at selected times during the cooling process. Lower panel: hadron local energy spread profiles at the same times. As in Fig. 6, we include data with and without cooling.

\section{ADDING INTRABEAM SCATTERING}

An important source of diffusion in a storage ring is intrabeam scattering (IBS) arising out of small-angle Coulomb collisions between the hadrons. In this section, we seek to incorporate this effect into our formalism in a simplified, phenomenological fashion. To begin with, we note that the additional contribution to the diffusion coefficient due to IBS is proportional to the hadron current $I_{h}$ [12]. As a result, this contribution can be expressed as

$$
D_{\mathrm{IBS}}=\frac{\sigma_{h, 0}^{2}}{\tau_{\mathrm{IBS}}} \frac{I_{h}}{I_{h, 0}}
$$

where $\tau_{\text {IBS }}$ is a measure of the IBS timescale. Written in this form, the above quantity represents the local diffusion rate due to IBS, which depends on the local current $I_{h}(z, \tilde{t})$ along the hadron beam. To switch from this local quantity to its $\phi$-averaged counterpart, we follow the same procedure as before. In particular, the equivalent to Eq. (15) is

$$
\tilde{D}_{\mathrm{IBS}}(\tilde{J}, \tilde{t})=\frac{1}{\pi} \int_{-\pi}^{\pi} d \phi \frac{\tau_{s} D_{\mathrm{IBS}}(\tilde{J}, \phi, \tilde{t})}{\sigma_{h, 0}^{2}} \sin ^{2} \phi
$$

where $D_{\mathrm{IBS}}(\tilde{J}, \phi, \tilde{t}) \rightarrow\left(\sigma_{h, 0}^{2} / \tau_{\mathrm{IBS}}\right) I_{h}(\sqrt{2 \tilde{J}} \cos \phi, \tilde{t})$. Combining these relations with the expression of Eq. (17) for the hadron current ratio, we obtain

$\tilde{D}_{\mathrm{IBS}}(\tilde{J}, \tilde{t})=\frac{2 \tau_{s}}{\pi \tau_{\mathrm{IBS}}} \frac{\int_{0}^{\pi} d \phi \sin ^{2} \phi \int_{0}^{\infty} d \tilde{\eta} \bar{F}_{h}\left(\tilde{\eta}^{2} / 2+\tilde{J} \cos ^{2} \phi, \tilde{t}\right)}{\int_{0}^{\infty} d \tilde{\eta} \bar{F}_{h}\left(\tilde{\eta}^{2} / 2,0\right)}$,

with the total kinetic equation being

$\frac{\partial \bar{F}_{h}}{\partial \tilde{t}}=\frac{\partial}{\partial \tilde{J}}\left(\tilde{\mathcal{L}}(\tilde{J}) \bar{F}_{h}\right)+\frac{\partial}{\partial \tilde{J}}\left(\tilde{J}\left\{\xi(\tilde{t}) \tilde{D}_{0}(\tilde{J})+\tilde{D}_{\mathrm{IBS}}(\tilde{J}, \tilde{t})\right\} \frac{\partial \bar{F}_{h}}{\partial \tilde{J}}\right)$.

Just like Eq. (20) the above equation can also be solved numerically in an iterative way.

With the updated model in place, we solve the kinetic equation for the eRHIC parameters assuming an IBS timescale of about $1 \mathrm{~h}$ (which roughly matches the initial cooling rate). The results of our simulation run are summarized in Figs. 5-7. In the first of these three figures we plot the IBS diffusion term as a function of the action at different times, establishing that it is much less sharplypeaked than the original diffusion term $\tilde{D}_{0}(\tilde{J})$ (though 
comparison with Fig. 3 also shows that their peak values are of the same order of magnitude). This, of course, is just another manifestation of the fact that the bunch length of the cooler electron beam is much shorter than that of the hadron beam. As far as Figs. 6-7 are concerned, the lefthand side subplots refer to the full case with cooling and IBS, while their right-hand side counterparts correspond to a scenario where IBS is not mitigated by any cooling. Moreover, in Fig. 6 we are concerned with the evolution of the longitudinal emittance and peak current of the proton beam as it is being circulated through the MBEC lattice (also including snapshots of the distribution function), whereas Fig. 7 shows how the proton current and local energy spread profiles change with time.

For the full case (cooling and IBS) we note that a rapid increase of the peak current by almost a factor of two within two hours is followed by a long plateau and an eventual slight decrease. At the same time, the core longitudinal emittance (defined for the part of the beam with $95 \%$ of the total charge) eventually drops to about $40 \%$ of its starting value, while the full emittance starts growing steadily after an early reduction, eventually surpassing its initial value after five hours (an indicator of tail heating). Reflecting the above trends, the proton current profile becomes sharper with time (though not quite as sharp as in Fig. 4), while the cooling of the core part of the beam is again accompanied by heating of the tail regions. Because of the additional amount of diffusion due to intrabeam scattering the stabilized values for the peak current and core emittance are (respectively) smaller and larger compared to the case with no IBS. On the other hand, the contrast with the results that include only IBS is striking. Without cooling, both values of the emittance grow with time, with the peak current decreasing and the proton bunch becoming steadily broader. As expected, unmitigated IBS leads to heating all along the proton bunch, though not at a uniform rate (the heating being more intense near the current peak). Overall, this preliminary, qualitative result appears to support the notion that MBEC can effectively counteract IBS in order to preserve the necessary beam quality for a typical eRHIC configuration, at least as far as the core part of the bunch is concerned. Lastly, we should note that the tail heating effect can cause issues with beam lifetime. Indeed, a numerical calculation shows that as much as $7 \%$ of the bunch population is knocked out of the cooling region $(\tilde{J}<4)$ after $5 \mathrm{~h}$. A more detailed analysis would be required in order to accurately link this effect to possible beam loss due to halo formation.

\section{SUMMARY}

In this paper we have developed a formalism that covers the long-term ion beam evolution in microbunched electron cooling, including the key effects of synchrotron oscillations and intra-beam scattering (IBS). A kinetic equation is derived for the ion distribution function, which can be solved numerically in an iterative fashion. This approach has been benchmarked with a simplified test case that yields an analytical solution for the distribution function. We have used the resulting numerical algorithm to study a hypothetical MBEC cooler for the parameters of eRHIC. The main conclusion is that the cooling of the longitudinal phase space of the proton beam is accompanied by a bunch compression effect, along with a corresponding peak current boost. Additionally, while the core part of the compressed bunch (which contains the bulk of the charge) undergoes substantial cooling, this benefit can sometimes be accompanied by appreciable heating of the tail ends of the beam. At any rate, the available degree of cooling from MBEC appears, overall, to be sufficient to counteract the natural emittance growth due to IBS, an observation with potentially significant practical implications. Our analysis has disregarded transverse cooling for simplicity's sake, although an extension to incorporate that effect, along with synchro-betatron coupling, is possible. The same is true of shot noise effects in the electron beam, which-though neglected here-can also be included in our 1D framework.

\section{ACKNOWLEDGMENTS}

We would like to thank F. Willeke and M. Blaskiewicz for useful discussions and comments regarding the subject of this paper. This work was supported by the Department of Energy, Contract No. DE-AC02-76SF00515.

\section{APPENDIX A: COOLING TERM IN THE KINETIC EQUATION}

In view of Eq. (6), we start from the expressions for the derivatives with respect to $J$ and $\phi$ :

$$
\begin{gathered}
\frac{\partial}{\partial J}=\frac{1}{\sqrt{2 J}} \cos \phi \frac{\partial}{\partial z}+\frac{1}{\sqrt{2 J}} \sin \phi \frac{\partial}{\partial \eta}, \\
\frac{\partial}{\partial \phi}=-\sqrt{2 J} \sin \phi \frac{\partial}{\partial z}+\sqrt{2 J} \cos \phi \frac{\partial}{\partial \eta} .
\end{gathered}
$$

From these equations, we deduce that

$$
\eta \frac{\partial F_{h}}{\partial z}-z \frac{\partial F_{h}}{\partial \eta}=-\frac{\partial F_{h}}{\partial \phi}
$$

We can also find the derivative with respect to $\eta$ that is needed on the right-hand side of the cooling equation. For this case, we find

$$
\frac{\partial}{\partial \eta}=\sqrt{2 J} \sin \phi \frac{\partial}{\partial J}+\frac{1}{\sqrt{2 J}} \cos \phi \frac{\partial}{\partial \phi} .
$$

Neglecting the diffusion term for the moment, the kinetic equation takes the form 


$$
\frac{\partial F_{h}}{\partial t}-\frac{\partial F_{h}}{\partial \phi}=\frac{1}{2}\left(\sqrt{2 J} \sin \phi \frac{\partial}{\partial J}+\frac{1}{\sqrt{2 J}} \cos \phi \frac{\partial}{\partial \phi}\right)\left\{\nu(\sqrt{2 J} \cos \phi, \sqrt{2 J} \sin \phi) F_{h}\right\}
$$

We now average this equation over $\phi$ by applying the operator $(2 \pi)^{-1} \int_{-\pi}^{\pi} d \phi \ldots$ to both sides of the equation. This annihilates the second term on the left-hand side. Let us consider the first term on the right hand side, namely

$$
\begin{aligned}
\frac{1}{2} & \frac{1}{2 \pi} \int_{-\pi}^{\pi} d \phi \sqrt{2 J} \sin \phi \frac{\partial}{\partial J}\left\{\nu(\sqrt{2 J} \cos \phi, \sqrt{2 J} \sin \phi) F_{h}\right\} \\
\quad & \frac{1}{2} \frac{1}{2 \pi} \frac{\partial}{\partial J} \int_{-\pi}^{\pi} d \phi \sqrt{2 J} \sin \phi \nu(\sqrt{2 J} \cos \phi, \sqrt{2 J} \sin \phi) F_{h}-\frac{1}{2} \frac{1}{2 \pi} \int_{-\pi}^{\pi} d \phi \frac{1}{\sqrt{2 J}} \sin \phi \nu(\sqrt{2 J} \cos \phi, \sqrt{2 J} \sin \phi) F_{h} .
\end{aligned}
$$

Next, we consider the last term on the right-hand side of Eq. (A4), that is

$$
\frac{1}{2} \frac{1}{2 \pi} \int_{-\pi}^{\pi} d \phi \frac{1}{\sqrt{2 J}} \cos \phi \frac{\partial}{\partial \phi}\left\{\nu(\sqrt{2 J} \cos \phi, \sqrt{2 J} \sin \phi) F_{h}\right\}=\frac{1}{2} \frac{1}{2 \pi} \int_{-\pi}^{\pi} d \phi \frac{1}{\sqrt{2 J}} \sin \phi \nu(\sqrt{2 J} \cos \phi, \sqrt{2 J} \sin \phi) F_{h} .
$$

We see that Eq. (A6) cancels the last term in Eq. (A5). What is left is just the first term in Eq. (A5) and the kinetic equation becomes

$$
\frac{\partial \bar{F}_{h}}{\partial \tilde{t}}=\frac{1}{2} \frac{1}{2 \pi} \frac{\partial}{\partial J}\left(\sqrt{2 J} \int_{-\pi}^{\pi} d \phi \sin \phi \nu(\sqrt{2 J} \cos \phi, \sqrt{2 J} \sin \phi) F_{h}\right)
$$

where $\bar{F}_{h}(J, \tilde{t})=(2 \pi)^{-1} \int_{-\pi}^{\pi} F_{h} d \phi$ is the averaged distribution function. In the limit when $\omega_{s} \gg 1 / t_{c}$-where $t_{c}$ is the cooling time - the synchrotron motion makes the distribution function almost independent of $\phi$, so in this equation we can replace $F_{h}$ by $\bar{F}_{h}$ on the right-hand side. The resulting equation is

$$
\frac{\partial \bar{F}_{h}}{\partial \tilde{t}}=\frac{1}{\sqrt{2}} \frac{\partial}{\partial J}\left(\sqrt{J} \bar{\nu}(J) \bar{F}_{h}\right)
$$

where

$$
\bar{\nu}(J)=\frac{1}{2 \pi} \int_{-\pi}^{\pi} d \phi \nu(\sqrt{2 J} \cos \phi, \sqrt{2 J} \sin \phi) \sin \phi .
$$

Equation (A8) conserves the total number of particles. If integrated with weight $J$, it yields the rate of cooling for the average value of $J$.

\section{APPENDIX B: DIFFUSION TERM IN THE KINETIC EQUATION}

Next, we would like to repeat the derivation if we include a standard diffusion term of the form

$$
D(z) \frac{\partial^{2} F_{h}}{\partial \eta^{2}}
$$

To start with, we have the relations

$$
\frac{\partial}{\partial J} D=\frac{D^{\prime}}{\sqrt{2 J}} \cos \phi, \quad \frac{\partial}{\partial \phi} D=-D^{\prime} \sqrt{2 J} \sin \phi,
$$

where the prime denotes differentiation with respect to the single argument (in this case, z). First, in view of Eq. (A3), we write the right-hand side of Eq. (B1) as

$$
D\left(\sqrt{2 J} \sin \phi \frac{\partial}{\partial J}+\frac{1}{\sqrt{2 J}} \cos \phi \frac{\partial}{\partial \phi}\right)\left(\sqrt{2 J} \sin \phi \frac{\partial F_{h}}{\partial J}+\frac{1}{\sqrt{2 J}} \cos \phi \frac{\partial F_{h}}{\partial \phi}\right) .
$$

There will be 3 terms here and we need to average them over $\phi$. The first one is when we take the first term from the first bracket and the first term from the second one, yielding 


$$
\begin{aligned}
I_{1} & =D \sqrt{2 J} \sin \phi \frac{\partial}{\partial J}\left(\sqrt{2 J} \sin \phi \frac{\partial F_{h}}{\partial J}\right) \\
& =2 \frac{\partial}{\partial J} J D \sin ^{2} \phi \frac{\partial F_{h}}{\partial J}-\sqrt{2 J} \sin ^{2} \phi \frac{\partial F_{h}}{\partial J} \frac{\partial}{\partial J}[D \sqrt{2 J}] \\
& =2 \frac{\partial}{\partial J}\left(D J \sin ^{2} \phi \frac{\partial F_{h}}{\partial J}\right)-\sqrt{2 J} \sin ^{2} \phi \frac{\partial F_{h}}{\partial J}\left(\frac{\partial D}{\partial J} \sqrt{2 J}+D \frac{1}{\sqrt{2 J}}\right) \\
& =2 \frac{\partial}{\partial J}\left(D J \sin ^{2} \phi \frac{\partial F_{h}}{\partial J}\right)-\sqrt{2 J} \sin ^{2} \phi \frac{\partial F_{h}}{\partial J}\left(D^{\prime} \cos \phi+D \frac{1}{\sqrt{2 J}}\right) .
\end{aligned}
$$

The second term is when we take the first term in the first bracket of Eq. (B3) and apply it to the second term in the second bracket. The end result is

$$
\begin{aligned}
I_{2} & =D \sqrt{2 J} \sin \phi \cos \phi \frac{\partial}{\partial J}\left(\frac{1}{\sqrt{2 J}} \frac{\partial F_{h}}{\partial \phi}\right)=D \sqrt{2 J} \sin \phi \cos \phi \frac{\partial}{\partial \phi} \frac{\partial}{\partial J}\left(\frac{F_{h}}{\sqrt{2 J}}\right) \rightarrow-\sqrt{2 J} \frac{\partial}{\partial J}\left(\frac{F_{h}}{\sqrt{2 J}}\right) \frac{\partial}{\partial \phi}(D \sin \phi \cos \phi) \\
& =\left(\frac{F_{h}}{2 J}-\frac{\partial F_{h}}{\partial J}\right)\left[-D^{\prime} \sqrt{2 J} \sin ^{2} \phi \cos \phi+D \cos ^{2} \phi-D \sin ^{2} \phi\right],
\end{aligned}
$$

where the arrow indicates a transformation that takes into account the averaging over $\phi$.

The third term is when we apply the second term in the first bracket of Eq. (B3) to the second bracket, which yields

$$
\begin{aligned}
I_{3} & =D \frac{1}{\sqrt{2 J}} \cos \phi \frac{\partial}{\partial \phi}\left(\sqrt{2 J} \sin \phi \frac{\partial}{\partial J}+\frac{1}{\sqrt{2 J}} \cos \phi \frac{\partial}{\partial \phi}\right) F_{h} \rightarrow-\frac{1}{\sqrt{2 J}}\left(\sqrt{2 J} \sin \phi \frac{\partial F_{h}}{\partial J}+\frac{1}{\sqrt{2 J}} \cos \phi \frac{\partial F_{h}}{\partial \phi}\right) \frac{\partial}{\partial \phi}(D \cos \phi) \\
& =-\frac{1}{\sqrt{2 J}}\left(\sqrt{2 J} \sin \phi \frac{\partial F_{h}}{\partial J}+\frac{1}{\sqrt{2 J}} \cos \phi \frac{\partial F_{h}}{\partial \phi}\right)\left[-D^{\prime} \sqrt{2 J} \sin \phi \cos \phi-D \sin \phi\right] .
\end{aligned}
$$

We now add up all terms contained in $I_{1}, I_{2}$, and $I_{3}$, the result being

$$
\begin{aligned}
2 & \frac{\partial}{\partial J}\left(D J \sin ^{2} \phi \frac{\partial F_{h}}{\partial J}\right)+\frac{F_{h}}{2 J}\left[-D^{\prime} \sqrt{2 J} \sin ^{2} \phi \cos \phi+D \cos ^{2} \phi-D \sin ^{2} \phi\right]-\frac{\partial F_{h}}{\partial J}\left[-D^{\prime} \sqrt{2 J} \sin ^{2} \phi \cos \phi+D \cos ^{2} \phi\right] \\
& +\left(\frac{1}{2 J} \cos \phi \frac{\partial F_{h}}{\partial \phi} D^{\prime} \sqrt{2 J} \sin \phi \cos \phi\right)+D \sin \phi\left(\sin \phi \frac{\partial F_{h}}{\partial J}+\frac{1}{2 J} \cos \phi \frac{\partial F_{h}}{\partial \phi}\right) .
\end{aligned}
$$

We can discard the terms that contain $\partial F_{h} / \partial \phi$-as this term vanishes when we replace $F_{h} \rightarrow \bar{F}_{h}$. Hence we are left with $2 \frac{\partial}{\partial J}\left(D J \sin ^{2} \phi \frac{\partial F_{h}}{\partial J}\right)+\frac{F_{h}}{2 J}\left[-D^{\prime} \sqrt{2 J} \sin ^{2} \phi \cos \phi+D \cos ^{2} \phi-D \sin ^{2} \phi\right]-\frac{\partial F_{h}}{\partial J}\left[-D^{\prime} \sqrt{2 J} \sin ^{2} \phi \cos \phi+D \cos ^{2} \phi-D \sin ^{2} \phi\right]$.

The expression in square brackets is equal to a total angle derivative, namely

$$
\frac{\partial}{\partial \phi}(D \sin \phi \cos \phi)
$$

so when we replace $F_{h} \rightarrow \bar{F}_{h}$ and integrate over $\phi$, this term disappears and we obtain only the first term in Eq. (B8). To recapitulate, $\phi$-averaging the original diffusion term yields the contribution

$$
\frac{\partial}{\partial J}\left(\bar{D}(J) J \frac{\partial \bar{F}_{h}}{\partial J}\right)
$$

to the right-hand side of the kinetic equation, where

$$
\bar{D}(J)=\frac{1}{\pi} \int_{-\pi}^{\pi} d \phi D(\sqrt{2 J} \cos \phi) \sin ^{2} \phi .
$$


[1] Y. S. Derbenev, AIP Conf. Proc. 253, 103 (1992).

[2] V. N. Litvinenko and Y. S. Derbenev, Phys. Rev. Lett. 102, 114801 (2009).

[3] D. Ratner, Phys. Rev. Lett. 111, 084802 (2013).

[4] M. Dohlus, E. A. Schneidmiller, and M. V. Yurkov, Phys. Rev. Accel. Beams 14, 090702 (2011).

[5] G. Stupakov, Phys. Rev. Accel. Beams 21, 114402 (2018).

[6] G. Stupakov and P. Baxevanis, Phys. Rev. Accel. Beams 22, 034401 (2019).

[7] P. Baxevanis and G. Stupakov, Phys. Rev. Accel. Beams 22, 081003 (2019).

[8] G. Wang, Phys. Rev. Accel. Beams 22, 111002 (2019).
[9] G. Stupakov, in IPAM Workshop on Beam Dynamics (2017).

[10] S. Y. Lee, Accelerator Physics (World Scientific, Singapore, 1999).

[11] P. Baxevanis and G. Stupakov, in Proceedings of the 2019 NAPAC Conference, WEPLH17 (Lansing, MI, USA, 2019).

[12] Handbook of accelerator physics and engineering, 2nd ed., edited by A. W. Chao, K. H. Mess, M. Tigner, and F. Zimmermann (World Scientific, Singapore, 2013), p. 155 . 